hemaglutinación, la neutralización y la virulencia. En cuanto a la infectividad viral, la VP4 debería dividirse en VP5 y VP8. ${ }^{14}$ La unión del rotavirus a los receptores que contienen ácido siálico facilita la penetración del virus en la célula. El primer contacto se establece a través de la VP8. ${ }^{14}$ Los antígenos del grupo ABO están presentes en los eritrocitos y en las células epiteliales. ${ }^{15}$ Al igual que el ácido siálico en el rotavirus VP8 animal, el rotavirus VP8 humano se une a los antígenos del grupo sanguíneo A en la misma localización. ${ }^{15}$ Sin embargo, en el estudio de Yazgan y colaboradores, ${ }^{16}$ no se ha establecido una asociación entre los grupos sanguíneos $\mathrm{ABO}$ y el rotavirus.

\section{CONCLUSIONES}

El grupo sanguíneo A se detecta con mayor frecuencia en los lactantes que tienen infección por rotavirus. Además, se ha determinado que las tasas de hospitalización de estos pacientes con grupo sanguíneo A son más altas. Por lo tanto, en los niños con el grupo sanguíneo A, podría ser necesario estudiar cuidadosamente la gastroenteritis por rotavirus.

\section{BIBLIOGRAFÍA}

1. DesselbergerU, Wolleswinkel-van den BoschJ,Mrukowicz J, Rodrigo C, et al. Rotavirus types in Europe and their significance for vaccination. Pediatr Infect Dis J 2006;25(1 Suppl):S30-41.

2. Kapikian A, Hoshino Y, Chanock RM. Rotaviruses. En: Knipe DM, Howley PM, Griffin DE, Lamb RA, et al, eds. Fields Virology. 4th ed. Philadelphia: Lippincott Williams \& Wilkins; 2001:1787-833.
3. Parez N. Rotavirus gastroenteritis: why to back up the development of new vaccines? Comp Immunol Microbiol Infect Dis 2008;31(2-3):253-69.

4. Gray J, Vesikari T, Van Damme P, Giaquinto C, et al. Rotavirus. J Pediatr Gastroenterol Nutr 2008;46(Suppl2):S2431.

5. Soriano-Gabarro M,MrukowiczJ, Vesikari T, Verstraeten T. Burden of rotavirus disease in European Union countries. Pediatr Infect Dis J 2006;25(1 Suppl):S7-11.

6. Giaquinto C, Van DammeP,HuetF, Gothefors L, et al. Costs of community-acquired pediatric rotavirus gastroenteritis in 7 European countries: the REVEAL Study. J Infect Dis 2007;195(Suppl 1):S36-44.

7. Ilver D, Arnqvist A, Ögren J, Frick IM, et al. Helicobacter pylori adhesin binding fucosylated histo-blood group antigens revealed by retagging. Science 1998;279:373-7.

8. Glass RI, Parashar UD, Estes MK. Norovirus gastroenteritis. N Engl J Med 2009;361(18):1776-85.

9. Clark B, McKendrick M. A review of viral gastroenteritis. Curr Opin Infect Dis 2004;17(5):461-9.

10. Velázquez FR, Matson DO, Calva JJ, Guerrero L, et al. Rotavirus infection in infants as protection against subsequent infections. N Engl J Med 1996;335(14):1022-8.

11. Bishop RF, Barnes GL,CiprianiE, Lund JS. Clinical immunity afterneonatal rotavirusinfection. A prospectivelongitudinal study in young children. N Engl J Med 1983;309(2):72-6.

12. Dilek İ, Demir C, Bay A, Akdeniz H, Öner A. ABO and Rh blood groups frequency in men and women living in Eastern Turkey. UHOD 2006;16(1):23-6.

13. Balci YI, Ovet G, Covut I, Goncu F, Yilmaz M. ABO and Rh blood groups frequency in Denizli Province. UHOD 2010;20(2):103-5.

14. Crawford SE, Mukherjee SK, Estes MK, Lawton JA, et al. Trypsin cleavage stabilizes the rotavirus VP4 spike. J Virol 2001;75(13):6052-61.

15. Liu Y, Huang P, Tan M, Liu Y, et al. Rotavirus VP8: phylogeny, host range, and interaction with histo-blood group antigens. J Virol 2012;86(18):9899-910.

16. Yazgan H, Keleş E, Gebeşçe A, Demirdöven M, Yazgan Z. Blood groups and rotavirus gastroenteritis. Pediatr Infect Dis J 2013;32(6):705-6.

\title{
Tratamiento de la epilepsia refractaria con dieta de Atkins modificada
}

\section{Treatment of refractory epilepsy with modified Atkins diet}

\author{
Dra. María Magdalena Vaccarezza ${ }^{a}$ Dra. Marisol Vanesa Toma ${ }^{a}$, Dr. Juan David Ramos Guevarab, \\ Dra. Cecilia Griselda Diez ${ }^{c}$ y Dr. Guillermo Eduardo Agosta ${ }^{a}$
}

a. Servicio de Neurología Infantil. Hospital Italiano de Buenos Aires. CABA. Argentina.

b. División de Neuropediatría. Fundación Hospital de la Misericordia. Universidad Nacional de Colombia. Bogotá, Colombia.

c. Servicio de Clínica Pediátrica. Hospital Italiano de Buenos Aires. CABA. Argentina.

\author{
Correspondencia: \\ Dra. María Magdalena Vaccarezza: \\ maria.vaccarezza@hospitalitaliano.org.ar \\ Conflicto de intereses: Ninguno que declarar.
}

Recibido: 27-3-2013

Aceptado: 5-2-2014 


\section{RESUMEN}

La dieta de Atkins modificada (DAM) es una alternativa dietaria terapéutica en el tratamiento de la epilepsia fármaco-resistente. Consiste en una dieta con un aporte de $60 \%$ de grasas, $30 \%$ de proteínas y $10 \%$ de carbohidratos.

El objetivo es presentar una serie de 9 pacientes con diagnóstico de epilepsia refractaria de diferentes etiologías, que recibieron tratamiento con DAM en nuestro hospital.

En nuestro grupo de 9 pacientes, obtuvimos resultados similares a los publicados por otros autores, con buena adherencia, tolerancia y respuesta. Del total de pacientes, dos lograron una reducción en más del 90\% del número de crisis; cuatro, del 5090\%; dos, menos del 50\% de control; y solo uno no presentó respuesta a la DAM. Ningún paciente presentó aumento del número de crisis, y fue bien tolerada en todos los casos.

Palabras clave: epilepsia refractaria, dieta Atkins modificada, dieta cetogénica, tolerancia, eficacia.

http:/ /dx.doi.org/10.5546/aap.2014.348

\section{INTRODUCCIÓN}

La epilepsia refractaria es una condición que ocurre en aproximadamente el $30 \%$ de los pacientes con epilepsia. ${ }^{1}$ Se define por la falta de respuesta a dos regímenes terapéuticos (apropiados y bien tolerados), ya sea como monoterapia o en combinación, para lograr que el paciente quede libre de crisis en forma sostenida.

La dieta cetogénica clásica (DCC) es una alternativa dietética terapéutica que se utiliza desde hace más de 90 años en el tratamiento de la epilepsia fármaco-resistente. ${ }^{2}$ Esta tendría un efecto anticonvulsivo mediante mecanismos de acción específicos en neurotransmisores y metabolitos. ${ }^{3}$ La DCC es una dieta estricta, en la que el $90 \%$ del aporte energético proviene de las grasas, lo que lleva ocasionalmente a dificultades para su cumplimiento y adherencia. ${ }^{4}$

La dieta Atkins fue creada en los años setenta por el Dr. Robert Atkins para el tratamiento de la obesidad. Su formulación estaba dada por un elevado aporte de grasas y un aporte de hidratos de carbono variable según el peso alcanzado por cada paciente.

A fines de los 90, surgió la dieta de Atkins modificada (DAM), que consiste en una dieta con un aporte calórico adecuado distribuido de la siguiente manera: $60 \%$ de grasas, $30 \%$ de proteínas y $10 \%$ de carbohidratos, sin restricción proteica, lo que ofrece mayor facilidad en su realización y una mejor tolerancia. ${ }^{4-6}$ En el año 2008, se publicaron los resultados del primer estudio aleatorizado controlado, en el que se observó la eficacia de la dieta cetogénica como tratamiento de las epilepsias refractarias en la población pediátrica y en adultos. ${ }^{7}$

\section{DESCRIPCIÓN DE CASOS}

Se presenta una serie de casos de niños con epilepsia refractaria, que recibieron tratamiento con dieta de Atkins en nuestro hospital, entre enero de 2008 y diciembre de 2012.

Se analizaron las historias clínicas de 9 pacientes con epilepsia refractaria que recibieron DAM. Las características clínicas de los pacientes se muestran en la Tabla 1. Uno de los pacientes había iniciado DCC y había realizado un cambio a DAM por falta de tolerancia.

En todos los casos se realizó una evaluación antes del inicio de la dieta que incluyó historia nutricional, registro diario de tipo y frecuencia de crisis, valoración clínica con examen antropométrico y panel de laboratorio.

El seguimiento se realizó con controles programados al mes, 3 y 6 meses de iniciada la dieta, y se evaluó la tolerancia y la adherencia, así como el registro diario de crisis.

La medición de la eficacia al tratamiento se evaluó a los 6 meses del inicio de la dieta mediante el control clínico del número de crisis. Se determinó que la respuesta era 1) excelente: si el control de las crisis era completo $(100 \%)$; 2 ) muy buen control: disminución de más del $90 \%$ de las crisis; 3) buen control: disminución del 50-90\% en el número de las crisis; 4) control regular: $<50 \%$ de disminución en el número de las crisis; 5) ausencia de efecto: sin cambio en el número de las crisis; y 6) negativo: aumento del número de las crisis. ${ }^{8}$

Aquellos pacientes que a los seis meses mostraron una reducción en el número de crisis mayor del $50 \%$ permanecieron en dieta durante dos años por considerarse efectiva para el control de crisis y fueron controlados de forma similar a los 12, 18 y 24 meses. Aquellos que no cumplían con esta condición interrumpieron la dieta de forma inmediata.

Del total de pacientes (9), dos lograron muy buen control de crisis; cuatro, buen control de crisis; dos, pobre control de crisis, y solo uno no presentó respuesta. Ningún paciente presentó empeoramiento con el inicio de la dieta. En la Tabla 1, se detallan para cada paciente las características de las crisis, así como la frecuencia absoluta de episodios previa al inicio de la DAM y a los 6 meses de haberla iniciado.

La respuesta evidenciada según el síndrome epiléptico fue:

- De los pacientes con crisis focales (seis): uno presentó muy buen control de crisis; tres, buenos controles de crisis; uno, control regular, y 
uno, ausencia de efecto; por lo tanto, no se observó diferencia significativa en el control de crisis dentro de este grupo.

- De los pacientes con síndrome de Lennox
Gastaut (dos): uno presentó muy buen control de crisis y otro, regular control.

- El paciente con síndrome de Dravet presentó buen control de crisis.

TABla 1. Características clínicas de la muestra

\begin{tabular}{|c|c|c|c|c|c|c|c|}
\hline Caso & $\begin{array}{l}\text { Edad } \\
\text { años/ } \\
\text { género }\end{array}$ & $\begin{array}{l}\text { Tipo } \\
\text { de crisis }\end{array}$ & Drogas & $\begin{array}{c}\text { Crisis mensuales } \\
\text { antes/después } \\
\text { de la DAM }\end{array}$ & $\begin{array}{c}\text { Disminución } \\
\text { (\%) del número } \\
\text { de crisis }\end{array}$ & $\begin{array}{c}\text { Efectos } \\
\text { adversos }\end{array}$ & $\begin{array}{l}\text { Duración } \\
\text { de la DAM }\end{array}$ \\
\hline 1 & 5 / Mujer & $\begin{array}{l}\text { - Focal motora } \\
\text { - Generalizada } \\
\text { (tónico/clónica) }\end{array}$ & $\begin{array}{c}\text { Topiramato } \\
\text { Levetiracetam } \\
\text { Ácido valproico }\end{array}$ & $240 / 90$ & $50-90 \%$ & No & 2 años \\
\hline 2 & 12 / Varón & $\begin{array}{l}\text { Generalizadas } \\
\text { - Atónicas } \\
\text { - Tónicas } \\
\text { - Ausencias } \\
\text { atípicas }\end{array}$ & $\begin{array}{l}\text { Clobazam } \\
\text { Lamotrigina } \\
\text { Topiramato } \\
\text { Levetiracetam } \\
\text { Rufinamida }\end{array}$ & $\begin{array}{c}60 / 40 \\
900 / 450\end{array}$ & $0-50 \%$ & $\begin{array}{c}\text { Hipokalemia } \\
\text { leve }\end{array}$ & 2 años \\
\hline 3 & 20 / Mujer & $\begin{array}{l}\text { - Focal motora } \\
\text { - Focal } 2^{\mathrm{a}} \text { generalizada }\end{array}$ & $\begin{array}{l}\text { Levetiracetam } \\
\text { Clobazam } \\
\text { Felbamato } \\
\text { Clobazam }\end{array}$ & $150 / 30$ & $50-90 \%$ & $\begin{array}{l}\text { Descenso } \\
\text { de peso } \\
\mathrm{IMC}=23, \\
\text { previo } 26\end{array}$ & $\begin{array}{l}\text { Continúa } \\
\text { (total: } \\
3 \text { años) }\end{array}$ \\
\hline 4 & 20 / Mujer & $\begin{array}{l}\text { Focal motoras } \\
\text { - Focal } 2^{\mathrm{a}} \text { generalizada }\end{array}$ & $\begin{array}{c}\text { Felbamato } \\
\text { Lamotrigina }\end{array}$ & $6 / 6$ & $0 \%$ & $\begin{array}{l}\text { Descenso } \\
\text { de peso } \\
\text { IMC = 19, } \\
\text { previo } 21\end{array}$ & 6 meses \\
\hline 5 & 21 / Varón & - Focal motoras & $\begin{array}{l}\text { Difenilhidantoína } \\
\text { Clobazam } \\
\text { Oxcarbazepina }\end{array}$ & $8 / 6$ & $0-50 \%$ & No & 6 meses \\
\hline 6 & 15 / Mujer & $\begin{array}{l}\text { Generalizadas } \\
\text { - Mioclónicas } \\
\text { - Ausencias } \\
\text { - Tónico-clónica }\end{array}$ & $\begin{array}{l}\text { Ácido valproico } \\
\text { Levetiracetam }\end{array}$ & $90 / 20$ & $50-90 \%$ & No & $\begin{array}{l}\text { Continúa } \\
\text { (total: } \\
1 \text { año) }\end{array}$ \\
\hline 7 & 15 / Mujer & $\begin{array}{l}\text { Generalizadas } \\
\text { - Ausencias atípicas } \\
\text { - Tónico-clónica }\end{array}$ & $\begin{array}{c}\text { Fenobarbital } \\
\text { Difenilhidantoína } \\
\text { Oxcarbazepina } \\
\text { Lamotrigina } \\
\text { Ácido valproico }\end{array}$ & $200 / 10$ & $+90 \%$ & No & $\begin{array}{l}\text { Continúa } \\
\text { (total: } \\
1 \text { año) }\end{array}$ \\
\hline 8 & 9 / Mujer & $\begin{array}{l}\text { - Focal motora } \\
\text { - Focal } 2^{\circ} \text { generalizado }\end{array}$ & $\begin{array}{c}\text { Lacosamida } \\
\text { Topiramato } \\
\text { Fenobarbital } \\
\text { Ácido valproico }\end{array}$ & $\begin{array}{r}150 / 5 \\
2 / 0\end{array}$ & $+90 \%$ & $\begin{array}{c}\text { Descenso } \\
\text { de peso } \\
\text { IMC }=17, \\
\text { revio 20, diarrea }\end{array}$ & $\begin{array}{l}\text { Continúa } \\
\text { (total: } \\
1 \text { año) }\end{array}$ \\
\hline 9 & 17 / Varón & - Focal motora & $\begin{array}{c}\text { Fenobarbital } \\
\text { Lamotrigina } \\
\text { Clobazam } \\
\text { Ácido valproico } \\
\text { Topiramato }\end{array}$ & $20 / 8$ & $50-90 \%$ & $\begin{array}{c}\text { Elevación del } \\
\text { colesterol }\end{array}$ & $\begin{array}{l}\text { Continúa } \\
\text { (total: } \\
1 \text { año) }\end{array}$ \\
\hline
\end{tabular}


De los 9 pacientes, 6 presentaron disminución de al menos $50 \%$ del número de crisis. Los pacientes que lograron reducción en el número de crisis continuaron sin modificaciones en los diferentes controles realizados a lo largo de los dos años de tratamiento con DAM.

Los efectos adversos encontrados fueron descenso de peso, hipercolesterolemia, diarrea esporádica autolimitada e hipokalemia. Estos consiguieron ser revertidos y en ningún caso condicionaron la interrupción de la dieta (ver la Tabla 1).

Cuatro pacientes se encuentran actualmente realizando la dieta y tres pacientes cumplieron dos años de tratamiento sin recaída al retirarla. Dos pacientes realizaron la dieta únicamente 6 meses ya que no tuvieron respuesta, y el cumplimiento de la dieta fue adecuado en todos los pacientes.

\section{DISCUSIÓN}

La dieta Atkins fue creada en los años setenta por el Dr. Robert Atkins para el tratamiento de la obesidad. Ante sus semejanzas con los principios de la DCC y siendo ésta menos rígida, en el centro de epilepsia pediátrica del Hospital John Hopkins, se iniciaron, en el año 2001, las primeras aproximaciones de su uso para epilepsia fármacoresistente.

Las primeras publicaciones en DAM datan de los años 2003 y 2006, ${ }^{5,7}$ en las cuales se presentaron series de casos que revelaron un efecto favorable en el control de crisis.

En 2006, ${ }^{5}$ un estudio prospectivo incluyó a 16 pacientes en tratamiento con DAM, con una media de edad de 6,5 años. Se evaluó el porcentaje de control de crisis a los 6 meses de haber iniciado la dieta: 12 pacientes presentaron una disminución de crisis de más del $50 \%$, y de estos, en 6, el control fue de más del $90 \%$.

En una reciente publicación de un estudio doble ciego de pacientes con epilepsia refractaria en los cuales se indicaba DAM, se evaluó su eficacia en niños. Los pacientes fueron enrolados por 3 meses en DAM y dieta normal en forma aleatorizada; posteriormente, los resultados mostraron que la dieta había sido efectiva en la reducción del número de crisis con escasos efectos adversos, con una diferencia estadísticamente significativa entre ambos grupos. ${ }^{9}$

Las diferencias entre la DCC y la DAM son, fundamentalmente, su composición nutricional y, por ende, su tolerabilidad. El aporte de grasas de la DAM es de un $60 \%$ del total, a diferencia de la
DCC, que es de un $90 \%$. Para el inicio de la DAM no se requiere internación a diferencia de la DCC. Las mediciones de los alimentos se realizan sobre la base de tablas de carbohidratos, sin necesidad de pesaje de alimentos, lo cual debe ser estricto en la DCC. El entrenamiento a los familiares para su implementación se realiza en consultas ambulatorias.

En la DAM, las proteínas son aportadas en forma libre, lo que permite evitar el incumplimiento de la dieta por falta de saciedad. La DAM puede ser una opción terapéutica para aquellos pacientes (adolescentes y adultos) con dificultades en la accesibilidad a un centro de dieta cetogénica o con alteraciones conductuales que impidieran el inicio o mantenimiento de la DCC (falta de saciedad, transgresión alimentaria, anorexia). . $^{8-13}$

La DAM podría ser considerada como segunda opción en aquellos pacientes que estuvieron en DCC con mala adherencia (pobre tolerancia a la restricción calórica) y cumplan, además, con alguno de los criterios previamente descriptos y en aquellos pacientes con encefalopatías epilépticas ${ }^{13}$ tales como síndrome de West, síndrome de Lennox Gastaut, síndrome de Dravet y epilepsias focales sintomáticas.

Para la implementación tanto de la DCC como de la DAM se requiere un equipo médico multidisciplinario especializado conformado por especialistas en nutrición y neurología, para un adecuado seguimiento de los pacientes y para lograr una buena adhesión al tratamiento.

En nuestro grupo, nueve pacientes obtuvieron resultados similares a los publicados por otros autores en términos de buena adherencia, tolerancia y control de crisis. Ninguno de los nueve pacientes presentó efectos adversos graves. En aquellos en los que la dieta fue efectiva, se pudo completar el tratamiento por dos años. Ningún paciente empeoró con el tratamiento.

Consideramos que la DAM debe ser tenida en cuenta como una opción de tratamiento, en especial en pacientes adolescentes y adultos con epilepsia refractaria.

\section{Agradecimiento}

Al Dr. Luis Panico, introductor/precursor en la instauración de este tratamiento en Argentina, por su desinteresada y paciente colaboración.

\section{BIBLIOGRAFÍA}

1. Kwan P, Arzimanoglou A, Berg A, Brodie MJ, et al. Definition of drug resistant epilepsy: consensus proposal 
by the ad hoc Task Force of the ILAE Commission on Therapeutic Strategies. Epilepsia 2010;51(6):1069-77.

2. Freeman JM, Vining EP, Kossoff EH, Pyzik PL, et al. A blinded, crossover study of the efficacy of the ketogenic diet. Epilepsia 2009;50(2):322-5.

3. Bough KJ, Rho JM. Anticonvulsant mechanisms of the ketogenic diet. Epilepsia 2007;48(1):43-58.

4. Kossoff EH, Krauss GL, McGroganJR, Freeman JM. Efficacy of the Atkins diet as therapy for intractable epilepsy. Neurology 2003;61(12):1789-91.

5. Kossoff EH, McGrogan JR, Bluml RM, Pillas DJ, et al. A modified Atkins diet is effective for the treatment of intractable pediatric epilepsy. Epilepsia 2006;47(2):421-4.

6. Sharma S, Sankhyan N, Gulati S, Agarwala A. Use of the modified Atkins diet for treatment of refractory childhood epilepsy: A randomized controlled trial. Epilepsia 2013;54(3):481-6.

7. Neal EG, Chaffe H, Shawartz RH, Lawson MS, et al. The ketogenic diet for the treatment of childhood epilepsy: a randomised trial. Lancet Neurol 2008;7(6):500-6.
8. Kossoff EH, DorwardJL. The Modified Atkins Diet. Epilepsia 2008;49(suppl 8):37-41.

9. Lee PR, Kossoff EH. Dietary treatments for epilepsy: management guidelines for the general practitioner. Epilepsy Behav 2011;21(2):115-21.

10. Kossoff EH. Intractable childhood epilepsy: choosing between the treatments. Semin Pediatr Neurol 2011;18(3):145-9.

11. Miranda MJ, Mortensen M,PovisenJH, Nielsen H, Beniczky S. Danish study of a Modified Atkins diet for medically intractable epilepsy in children: Can we achieve the same results as with the classical ketogenic diet? Seizure 2011;20(2):151-5.

12. Auvin S. Should we routinely use modified Atkins diet instead of regular ketogenic diet to treat children with epilepsy? Seizure 2012;21(4):237-40.

13. Lee PR, Kossoff EH. Dietary treatments for epilepsy: management guidelines for the general practitioner. Epilepsy Behav 2011;21(2):115-21.

\title{
Diecinueve años de vigilancia de enfermedad invasiva neumocócica en un hospital pediátrico de Mendoza, Argentina
}

\author{
Nineteen-years of pneumococcal invasive disease surveillance in a children's \\ hospital in Mendoza, Argentina
}

\author{
Dr. Héctor José Abate ${ }^{a}$ Dra. Andrea Falaschi ${ }^{a}$, Bioq. Laura Balbib y Bioq. Beatriz García ${ }^{b}$
}

\section{RESUMEN}

Estudiamos 537 niños internados en el Hospital Dr. Notti, entre 1993 y 2011, con enfermedad invasiva neumocócica. La mediana de edad fue 19 meses ( $\mathrm{R}=0-192 \mathrm{~m}) ; 34,82 \%$ fueron $<1$ año y $23,46 \%, \geq 60$ meses. Predominaron neumonía con y sin derrame $(48,04 \%)$ y meningitis $(29,05 \%)$, con una letalidad de $6,14 \%$. El 56,86\% de los serotipos identificados fueron 14,5 y 1 . Mostraron sensibilidad a la penicilina el $99,74 \%$ de cepas no meníngeas y a la ceftriaxona, el $98,08 \%$ de cepas meníngeas. Los factores de riesgo en neumonía con derrame se asociaron a la edad $\geq 60$ meses, RR 1,47 (1,06-2,04), p 0,02, serotipos 5, RR $2,57(1,71-3,87)$, p 0,0001 y 1 RR $1,86(1,17-2,96)$, p 0,014 y en

a. Servicio de Infectología.

b. Servicio de Microbiología.

Hospital Pediátrico Dr. Humberto Notti.

Guaymallén. Mendoza. Argentina.

\section{Correspondencia:}

Dr. Abate, Héctor José: hjabate@gmail.com.

Conflicto de intereses: Ninguno que declarar.

Recibido: 31-7-2013

Aceptado: 12-3-2014 las meningitis, principalmente $\mathrm{a}<1$ año, RR 2,35 (1,87-3,06), $\mathrm{p}$ 0,0000 y serotipo 18C, RR 2,19 (1,3-3,7), p 0,024.

Conclusión. El Streptococcus pneumoniae representó un problema importante en menores de un año, en quienes predominó la meningitis y causó más de la mitad de las muertes, y en mayores de 60 meses, en los que prevalecieron neumonías con derrame. La mayoría fueron sensibles a la penicilina y a la ceftriaxona. Palabras clave: enfermedad invasiva neumocócica, serotipos, sensibilidad antibiótica, factores de riesgo.

http://dx.doi.org/10.5546/aap.2014.352

\section{INTRODUCCIÓN}

El Streptococcus pneumoniae (Spn) causa enfermedades inmunoprevenibles de alta mortalidad. ${ }^{1}$ Una revisión de datos en América Latina y el Caribe, entre 1990 y 2006, mostró que entre 12000 y 28000 menores de 5 años podrían morir anualmente a causa de esta enfermedad. ${ }^{2}$

Los serotipos responsables de la mayoría de las enfermedades invasivas neumocócicas (EIN) muestran variaciones geográficas y según el tipo de enfermedad, ${ }^{3}$ además de cambios en la susceptibilidad antimicrobiana, especialmente a la penicilina y a las cefalosporinas de tercera 